
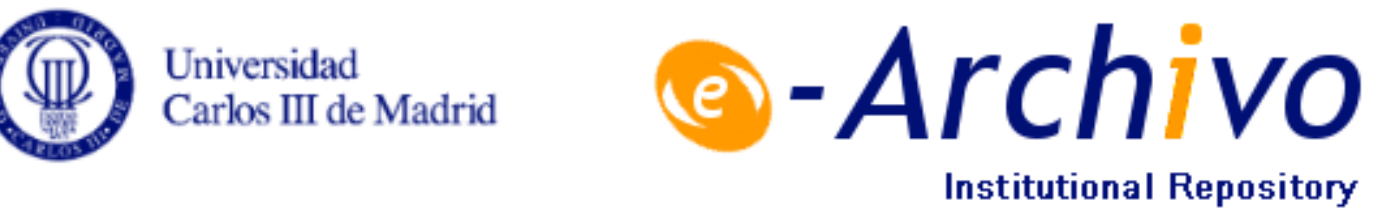

This is a postprint version of the following published document:

González-Benito, J., Mikeš, F., Baselga, J. \&

Lemetyinemm, H. (2002). Fluorescence method using labeled chromophores to study the curing kinetics of a polyurethane system. Journal of Applied Polymer Science, 86 (12), pp. 2992-3000.

DOI: 10.1002/app.11281

(C) Wiley, 2002 


\title{
Fluorescence Method Using Labeled Chromophores to Study the Curing Kinetics of a Polyurethane System
}

\author{
J. González-Benito, ${ }^{1}$ F. Mikeš, ${ }^{1}$ J. Baselga, ${ }^{1}$ H. Lemetyinemm ${ }^{2}$ \\ ${ }^{1}$ Department of Materials Science and Metallurgic Engineering, Universidad Carlos III de Madrid \\ ${ }^{2}$ Institute of Materials Chemistry, Tampere University of Technology, P.O. Box 541, FIN-33101, Tampere, Finland
}

\begin{abstract}
A fluorescence method using labeled chromophores to study the curing kinetics of a polyurethane (PU) system was developed. A PU system based on fluorescent-labeled hexamethylene diisocyanate and a polyol (polyether/polyester) was cured at different temperatures $\left(25,40,50\right.$, and $\left.65^{\circ} \mathrm{C}\right)$. The fluorescent response from the 5-

dimethylaminonaphtalene-1-( $N$-2-aminoethyl)sulfonamide and 4-methacryloylamino-4'-nitrostilbene moieties chemically bonded to the PU system was monitored as a function of the curing time. With the fluorescence data, it was possible to model the kinetics of the curing process. Methods based on the fluorescence intensity ratio and the first moment of the fluorescent band emission were applied to determine the degree of curing of the PU system. In addition, it was possible to calculate an apparent activation energy for the curing process, and a value of $17 \mathrm{~kJ} / \mathrm{mol}$ was obtained.
\end{abstract}

\section{INTRODUCTION}

The polymerization processes of diisocyanate monomers are quite interesting because of the importance of the production of polyurethane (PU), which is used for high-performance materials such as coatings, elastomers, adhesives, foams, and structural composites. Therefore, a quantitative understanding of the reaction kinetics is essential for assessing the feasibility and design of commercial PU processes. A number of previous studies have focused on the mechanism and kinetics of these reactions, ${ }^{1-11}$ and they have reveled a number of different mechanisms. For instance, the mechanisms depend on the reaction mixture composition, temperature, moisture, catalysis, and so forth. In most cases, the kinetics of the reactions between isocyanate (NCO) and hydroxyl $(\mathrm{OH})$ groups has been analyzed according to the classical second-order reaction rate equation, ${ }^{7-11}$ although deviations from second-order kinetics have often been found. . $^{1,3,5,10,11}$ These deviations can be explained, according to the system and reaction conditions, in terms of the catalytic effects, secondary competing reactions, and gel formation. ${ }^{4,7}$ However, the mechanism is not clear at the moment. The establishment of a kinetic model and knowledge of the contributions of various kinetic pa-

Correspondence to: J. González-Benito (javid@ing.uc3m.es). Contract grant sponsors: Carlos III University; Tampere University of Technology. rameters, such as the activation energy, in complex reactions provide control over the production of final products with the desired handling properties.

There are a number of classical techniques used to investigate cure processes, such as differential scanning calorimetry (DSC), dynamic dielectric analysis, dynamic mechanical thermal analysis, and Fourier transform infrared (FTIR). However, none of these have proven to be very sensitive, nondestructive techniques for monitoring in situ and real-time polymerization processes. The most common analytical methods for kinetic studies of the curing of PU systems are FTIR and DSC, although they show some limitations in sensitivity at high degrees of the curing process. In FTIR studies, the decrease in the NCO band at 2271 $\mathrm{cm}^{-1}$ is often used to monitor the curing process, ${ }^{10,12}$ although some difficulties appear when the analysis conditions are not very well controlled because the bending $\mathrm{CO}_{2}$ band can interfere with the NCO band. For DSC, the dynamic, isothermal, and model-free isoconversional modes have been used to monitor the curing process of PU systems. ${ }^{9}$ Conversions obtained from these three methods agree well with one another, indicating that the isothermal and model-free isoconversional modes can successfully be used for monitoring the curing process. ${ }^{9}$ Nevertheless, DSC methods are not suitable for monitoring real-time processes or for the nondestructive analysis of polymers.

Fluorescence is a very sensitive and nondestructive technique for monitoring the curing of different polymeric systems. ${ }^{13-22}$ The use of the fluorescent response from labels has become a very powerful tool for fol- 
lowing changes in its surroundings, such as the polarity and rigidity. ${ }^{13-25}$ Some studies have proposed that an enhancement in the microviscosity of the medium leads to a decrease in the nonradiative decay rate and, consequently, an increase in the fluorescence quantum yield. ${ }^{17}$ Others use the increase in the fluorescence intensity that comes from the chemical changes in the fluorophore. ${ }^{18,21}$ However, this method does not eliminate the effect of intensity variations arising from external factors, such as lamp intensity, optical alignment, probe location, excitation area, and temperature variation. Because of these limitations, new methods for fluorescence analysis are required. Neckers and coworkers $^{26,27}$ reported the use of fluorescence probes for monitoring the curing process of polyacrylate monomers with an intensity ratio method, and more recently, Lemmetyinen and coworkers ${ }^{19,20}$ developed a new intensity ratio method, in which the ratios of the low-to-high-intensity changes (LHICs) in the emission bands were used to determine the degree of the curing processes.

However, some fluorescent molecules, such as dansyl (DNS) 16,28,29 $^{2}$ and 4-dimethyamino-4'-nitrostilbene (DANS) derivatives, ${ }^{14,20}$ show an appreciable shift in their fluorescence emission band, depending on the polarity and rigidity of their surroundings. Therefore, their spectral shifts seem to be good photophysical parameters for monitoring the changes appearing in polymeric systems. In general, the dipole moment of fluorophores in the excited state is higher than the dipole moment in the ground state, especially for fluorescence moieties such as DNS and DANS derivatives. Because of this, in the excited state it is possible to establish an effective dielectric coupling between the dipole moment of the excited fluorophore and the dipole moments of the surrounding groups in the system. When the dielectric coupling occurs, a stabilization of the excited state appears, and more effective coupling leads to greater stabilization. This characteristic behavior is translated into a redshift in the fluorescence emission band of the fluorophore. ${ }^{28}$ Therefore, the position of the fluorescence emission will be affected by changes in the dielectric constant of the system and changes in the refractive index. However, the stabilization of the excited state of the fluorophore depends on the relaxation time of the surrounding dipoles. The stabilization of the excited state is only possible when that relaxation time is shorter than the lifetime of the fluorophore excited state. ${ }^{28}$ Therefore, the greater the relaxation times are, the less effective the dielectric coupling is and the smaller the redshift is. Because curing processes imply the enhancement of the viscosity in the system, there must be a decrease in the molecular motions, and so the relaxation times must be shorter; therefore, a blueshift of the fluorescence emission band is expected with the curing time.
Generally, changes in the value of the maximum in the emission band are measured as the wavelength or wavenumber. Nevertheless, when small shifts are involved, the spectra may not have sufficient resolution to detect variations without a large dispersion in the data. ${ }^{13}$ To solve this problem, we propose using the average of the wavenumber from the emission band, that is, the first moment, $\langle\nu\rangle$, defined as follows:

$$
\langle\nu\rangle=\frac{\sum \nu_{i} I\left(\nu_{i}\right)}{\sum I\left(\nu_{i}\right)}
$$

where $\nu_{i}$ is a particular wavenumber in the range of the fluorescence spectrum and $I\left(\nu_{i}\right)$ is the fluorescence intensity for that particular wavenumber.

Finally, with a bifurcate optical guide, it is possible both to excite the sample and to collect its fluorescence, nondestructively, in situ and even with online processes.

The aim of this work was to model the kinetics of the reaction of hexamethylene diisocyanate-based PU with new fluorescence conversion $\left(\alpha_{f}\right)$ data obtained from photophysical parameters. Two fluorescence labels, DNS and DANS (chemically attached to the diisocyanate component), were used to monitor the curing process of the $\mathrm{PU}$, and the intensity ratio and first-moment methods were applied to evaluate the reaction rate at any conversion.

\section{EXPERIMENTAL}

The PU system studied in this work was prepared by the mixing, at room temperature, of the polyol (polyether/polyester, $58.7 \mathrm{wt} \%$ ) with the accelerator (dibutyltin dialurate, $0.117 \mathrm{wt} \%$ ) and $\mathrm{NCO}$ (hexamethylene diisocyanate, $41.3 \mathrm{wt} \%$ ). After that, the system was cured at predetermined temperatures. This PU formulation, commercially named PU1096, was supplied by Gairesa Co. (Lugo, Spain).

Two reactive fluorescence molecules that showed spectral shifts with microviscosity changes were selected, 5-dimethylaminonaphtalene-1-( $N$-(2-aminoethyl)sulfonamide (DNS label) and 4-methacryloylamino-4'-nitrostilbene (DANS label), which were synthesized at Carlos III University (Madrid, Spain). Both of them had an amine functionality to react with the NCO groups. The labeling reactions were carried out by the mixing of the first component of the PU1096 system with the fluorescent moiety, stirring with a magnetic bar, and heating for $16 \mathrm{~h}$ at $60^{\circ} \mathrm{C}$. The amount of the fluorescence label was calculated to be $2.5 \times 10^{-4} \mathrm{~mol} / \mathrm{kg}$ of PU mixture.

For the preparation of the PU polymer samples, the labeled first component was mixed with the second component in a weight proportion of $1.42 / 1.0$. The mixtures were placed between two glass plates, their 


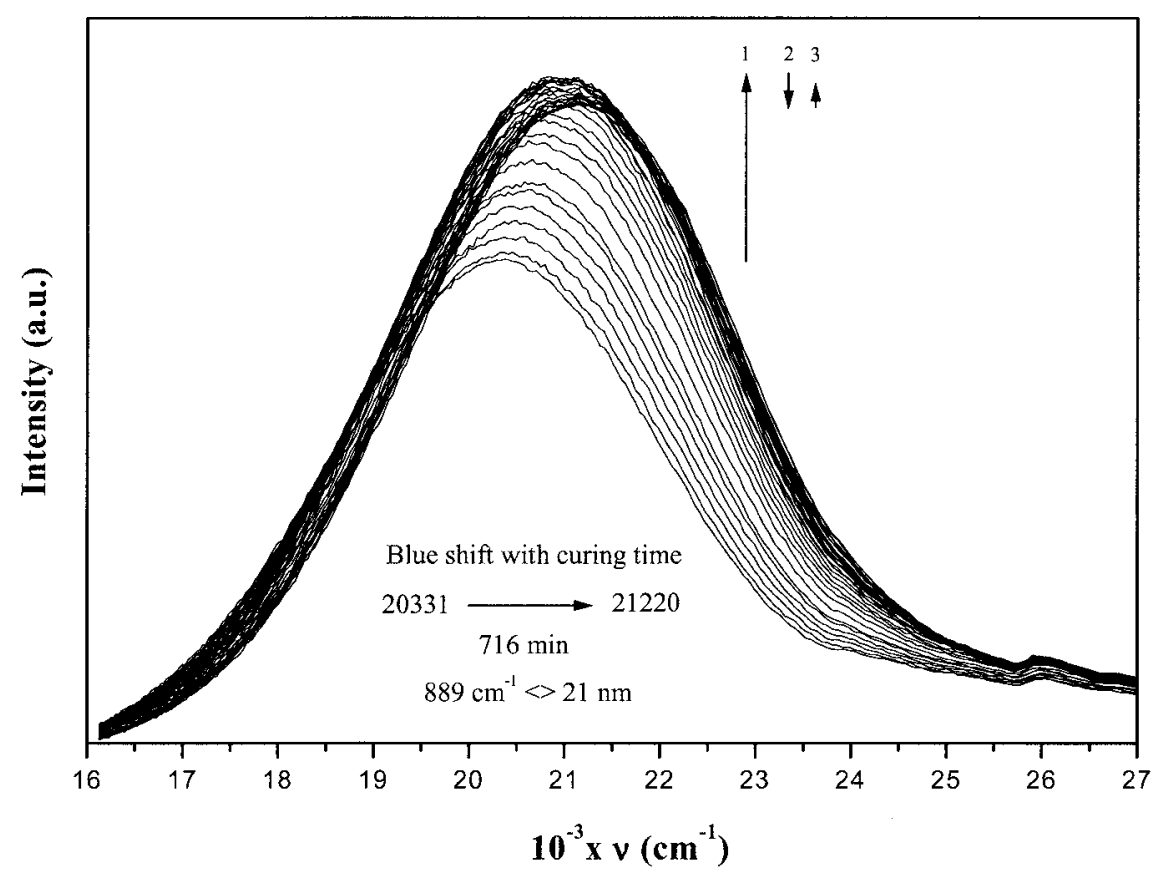

Figure 1 Fluorescence emission spectra of the DNS label during the curing of PU1096 at $25^{\circ} \mathrm{C}$. The vertical arrows indicate the order of spectra during the curing process.

thicknesses controlled with double-coated tape (0.9 $\mathrm{mm})$. The curing processes were monitored at four temperatures $\left(25,40,50\right.$, and $\left.65^{\circ} \mathrm{C}\right)$ with a Spex Fluorolog 3 spectrofluorometer with front-face geometry. The sample was placed in a typical sample holder with a thermostated jacket, and for control of the temperature at any moment, a thermocouple was mounted on the glass plates. The excitation wavelengths were 360 and $415 \mathrm{~nm}$, and the spectra were recorded automatically at regular intervals of time from 370 to $620 \mathrm{~nm}$ and from 425 to $700 \mathrm{~nm}$ for the DNS-labeled and DANS-labeled PU systems, respectively.

The experimental data were examined with an Excel macro developed by the authors that calculates from the spectra the integrated fluorescence intensity, the LHIC values, and the first-moment values.

\section{RESULTS AND DISCUSSION}

\section{DNS label}

As an example, in Figure 1 the fluorescence spectra of the DNS label are shown for different curing times at $25^{\circ} \mathrm{C}$. A broad band is observed that undergoes a blueshift when the curing of PU proceeds. In addition, the intensity clearly shows changes as a function of the curing time. In Figure 2, the evolution of the normalized integrated intensity of the DNS-labeled PU with the curing time at $25,40,50$, and $65^{\circ} \mathrm{C}$ is plotted.

The emission intensity of the DNS label shows similar tendencies. There is a general increase in the in- tensity as the curing of PU takes place at the temperature used for the reaction. However, some fluctuations in the intensity variations did not allow correlating the intensities directly with a conversion percentage. If we consider the interpretation of Loufty ${ }^{17}$ for the increase in the fluorescence intensity with an increase in the viscosity, the curing time for which the intensity starts leveling off might be considered the beginning of the vitrification region. Assuming the last argument, in Figure 2 we show the vitrification times, and they are, as expected, dependent on the temperature of curing.

Because the variation of the fluorescence intensity does not seem to be a good parameter for determining the degree of curing, LHICs ${ }^{19,20}$ and the first-moment ${ }^{13}$ methods have been used.

In the LHIC method, the spectrum of the cured polymer, measured at infinite time, is divided by the spectrum of the mixed monomers, measured at time zero. Therefore, the intensity changes are obtained as a function of the wavenumber, and the low-intensity changes and high-intensity changes can be found. The results for the DNS label are presented in Figure 3.

The LHIC curves are obtained by the intensities at a certain wave number in the shorter wave-number region $\left(16,863 \mathrm{~cm}^{-1}\right)$ being divided by the intensities at the longer wave-number region $\left(22,831 \mathrm{~cm}^{-1}\right)$ at all moments of the curing time. Normalized LHIC ratios of the DNS label as a function of the curing time of the PU1096 system for four temperatures are shown in Figure 4. 

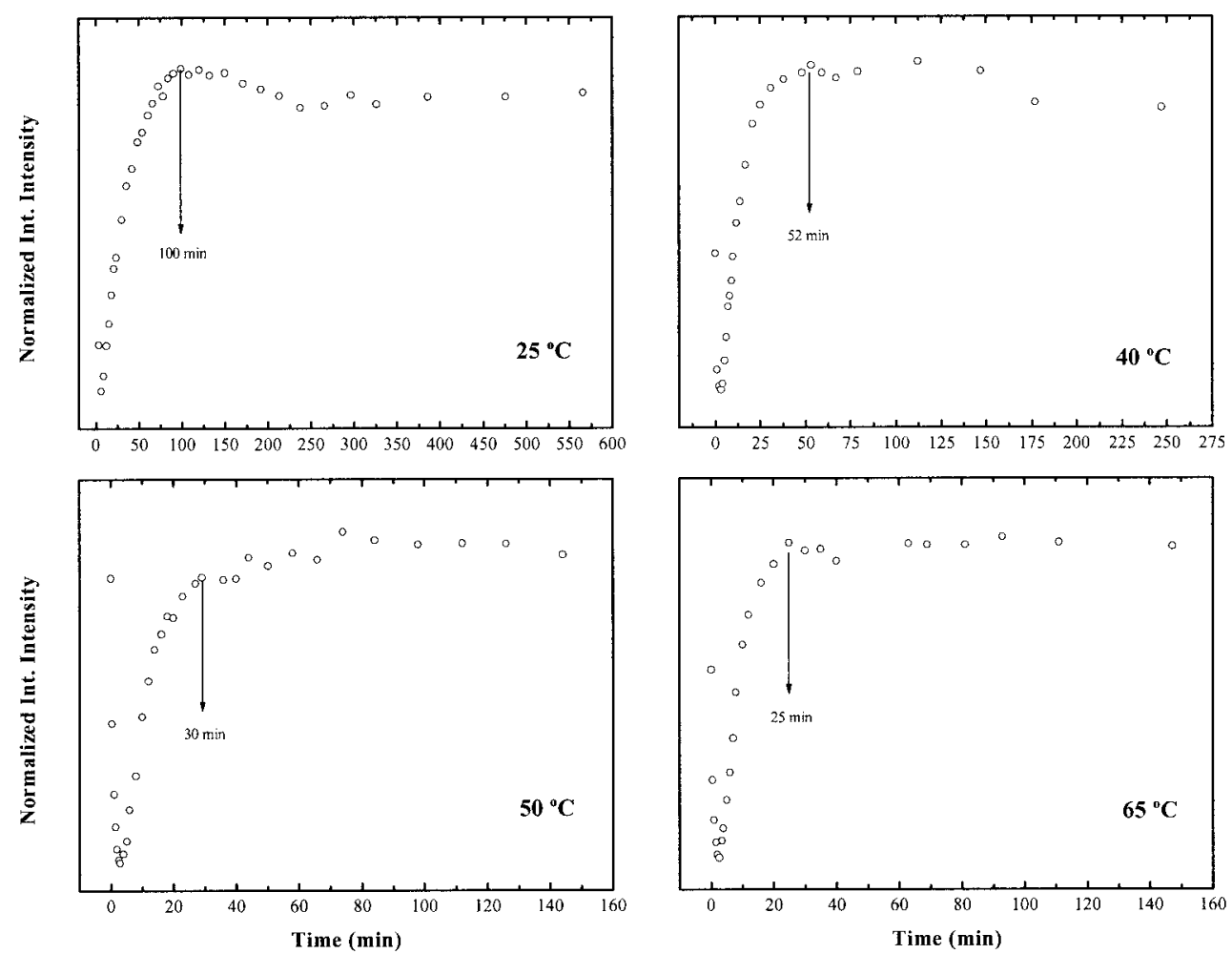

Figure 2 Evolution of the normalized integrated intensity of the DNS-labeled PU1096 with the curing time at 25, 40, 50, and $65^{\circ} \mathrm{C}$.

Correlations between the normalized LHIC ratios and conversion percentages measured by FTIR $^{10}$ are shown in Figure 5. As can be seen, although only at $25^{\circ} \mathrm{C}$ there is a nearly linear correlation, the plots clearly show evidence that this method is related to the conversion of the curing in PUs at any reaction time.

In the first-moment method, the energy average of the fluorescence emission spectra, $\langle\nu\rangle$, is obtained at different curing times. The $\langle\nu\rangle$ values of the DNS

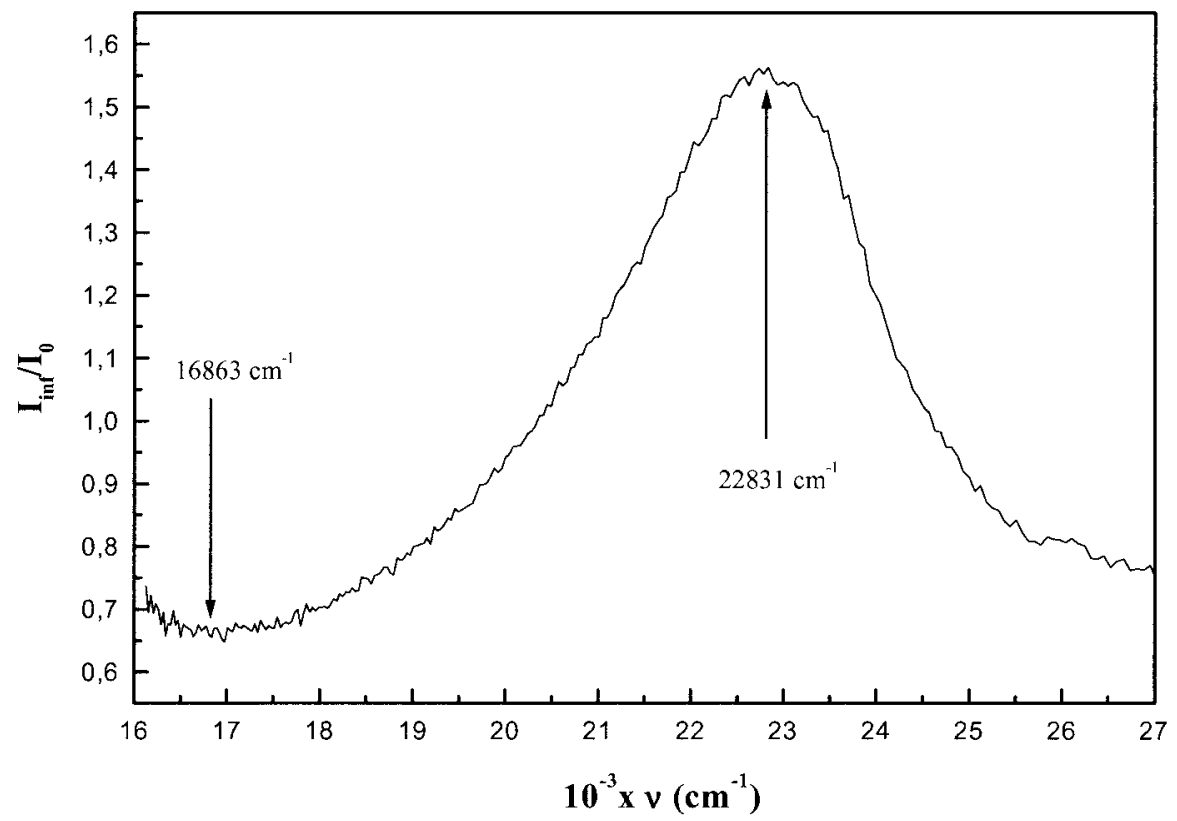

Figure 3 Ratio of the fluorescence intensities (infinite time/time zero) of the DNS label in the curing process of PU1096 as a function of the wave number. 

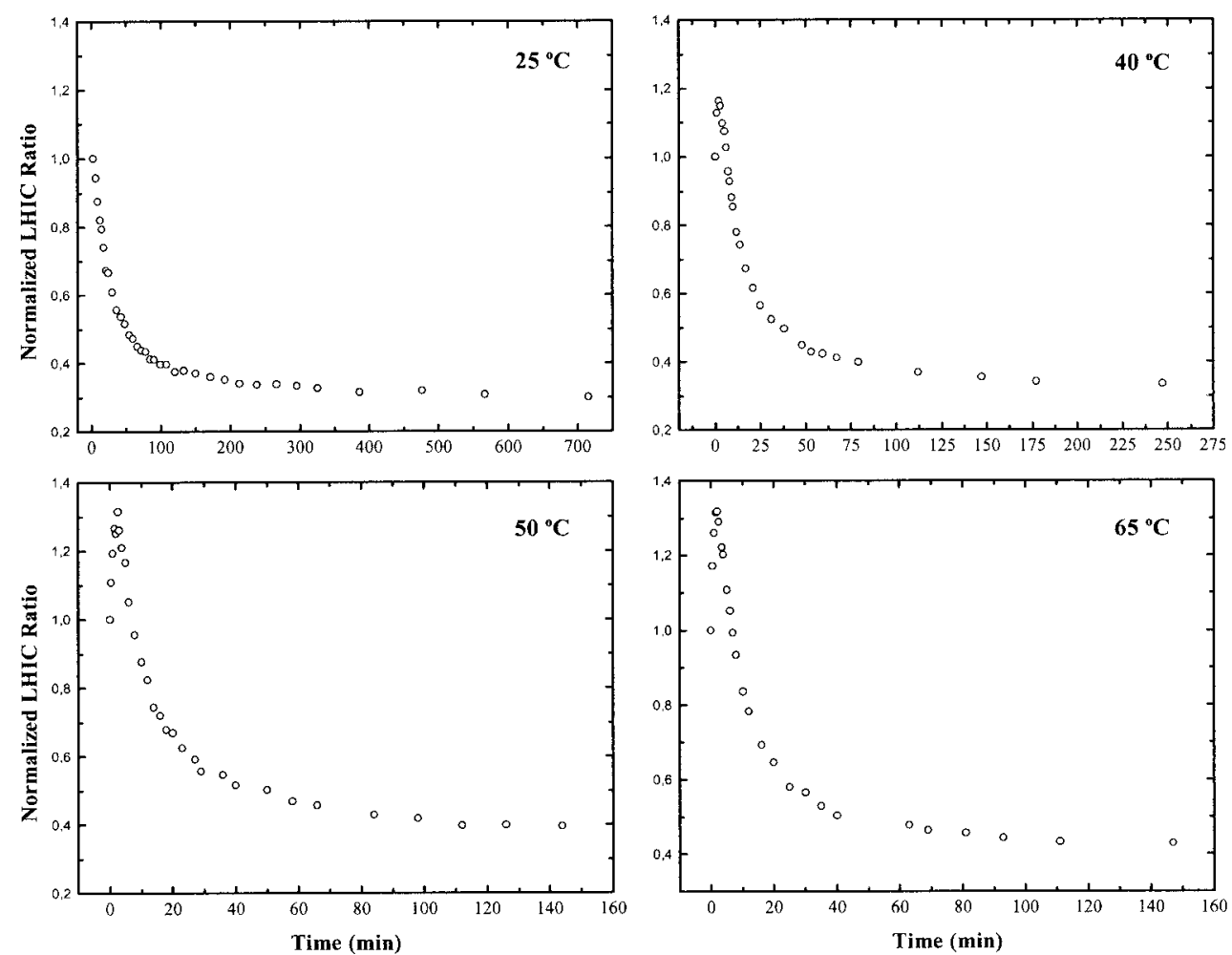

Figure 4 Normalized LHIC ratios of the DNS label as a function of the curing time of the PU1096 system for four temperatures.

label as a function of the curing time of the PU1096 system for four temperatures are shown in Figure 6. The general tendency at any curing temperature is that the $\langle\nu\rangle$ parameter increases as the reaction pro- ceeds. For the system under study, two large changes are happening during the cure process: (1) the polarity decreases as the $\mathrm{OH}$ groups are consumed and (2) the viscosity increases as the molec-

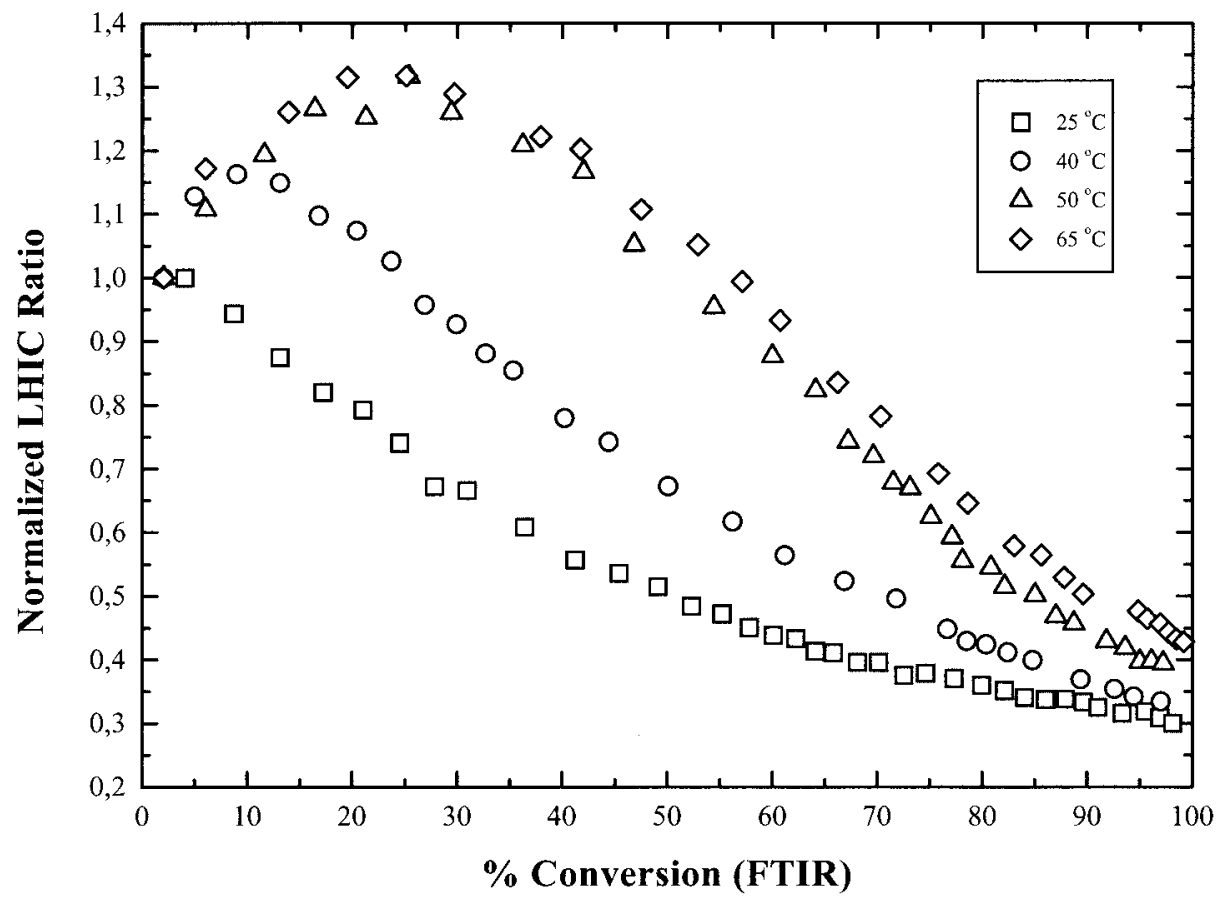

Figure 5 Normalized LHIC ratios as a function of the conversion obtained by FTIR for the PU1096 curing process. 

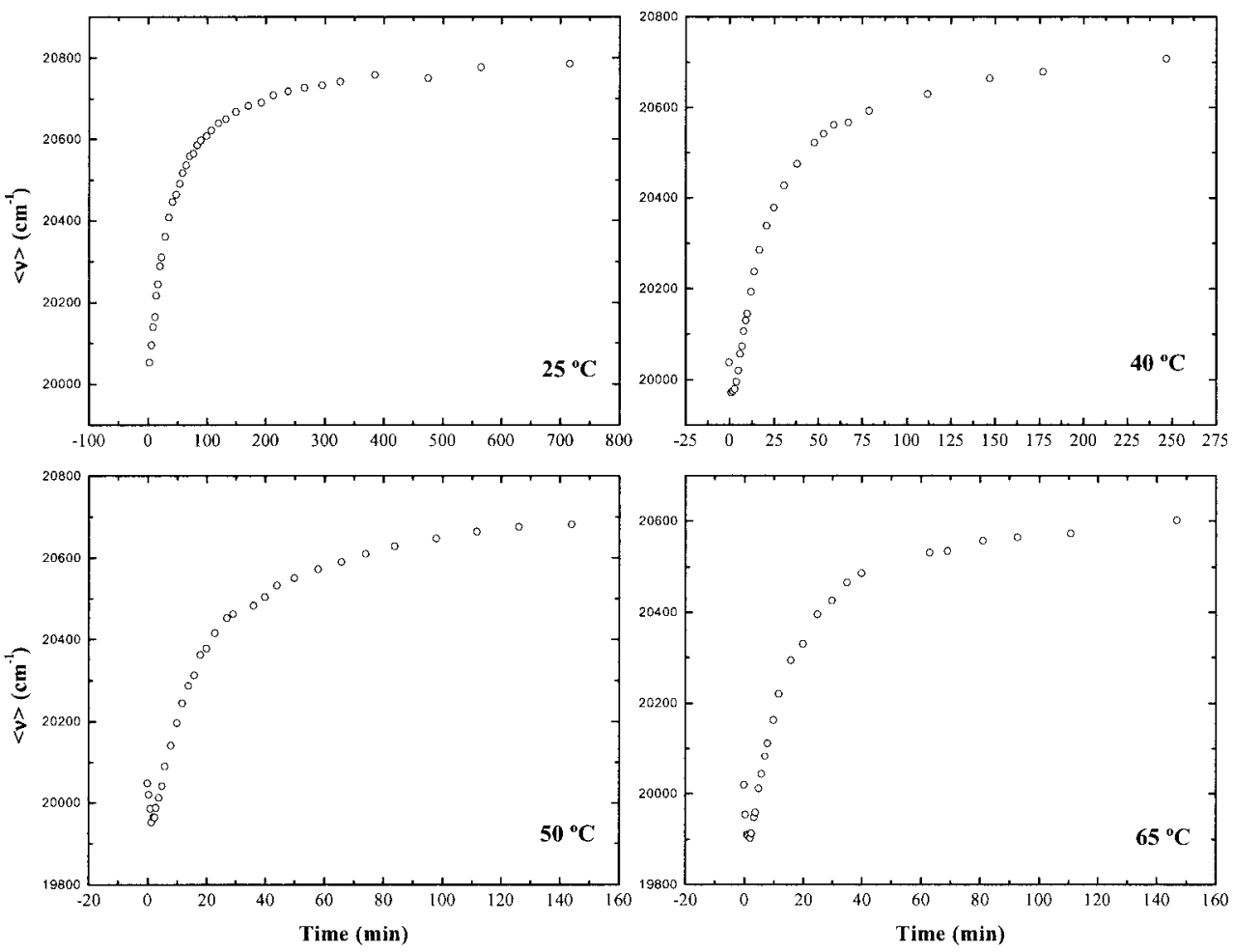

Figure 6 First-moment values, $\langle\nu\rangle$, of the fluorescence band (DNS label) as a function of the curing time of the PU1096 system for four temperatures.

ular weight and crosslink density increases. This result agrees with the blue Stokes shift expected in the emission of one fluorophore when the dipole moments of the surrounding groups are lower and when their motions are restricted. ${ }^{30}$ The selection of this parameter arises from the idea that the same variations occur for any fluorophore, although only when vibrational stabilizations are observed. In

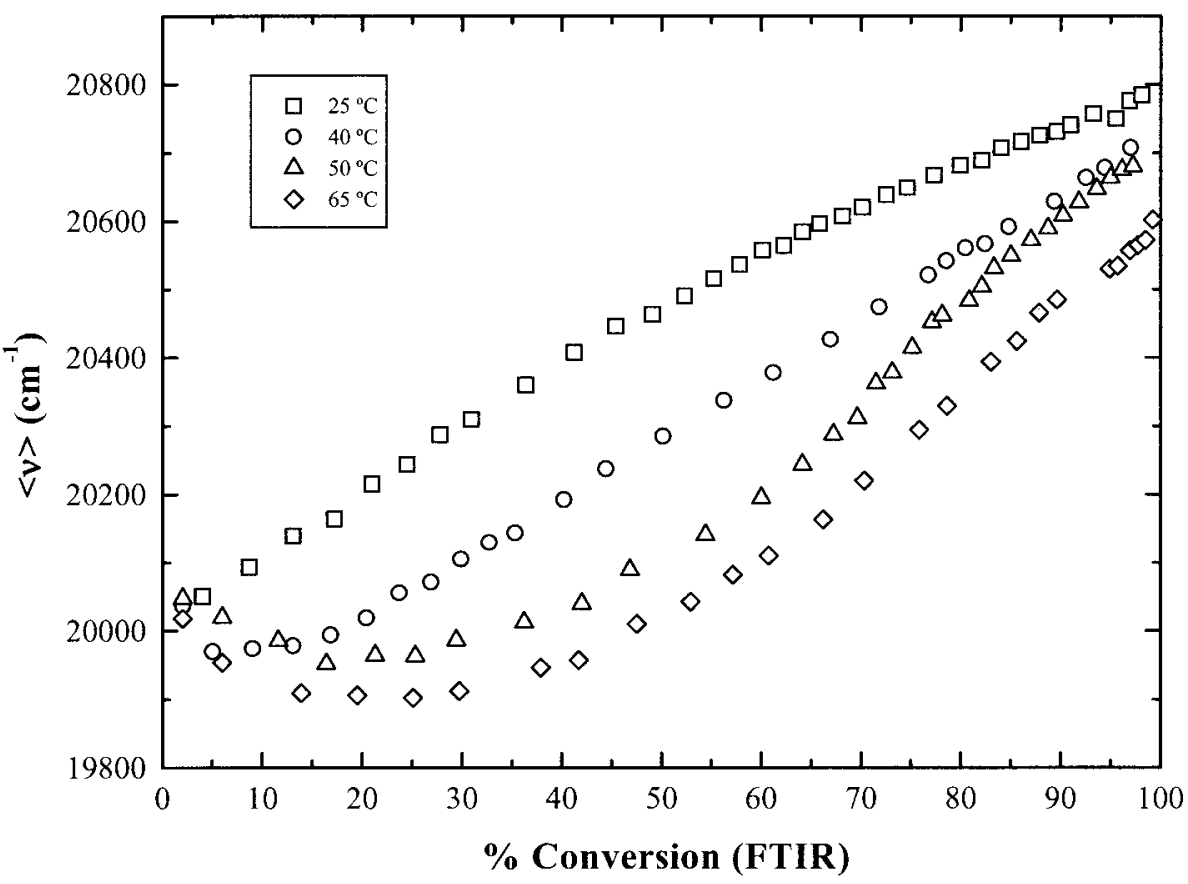

Figure 7 First-moment values, $\langle\nu\rangle$, of the fluorescence band (DNS label) as a function of the conversion obtained by FTIR for the PU1096 curing process. 


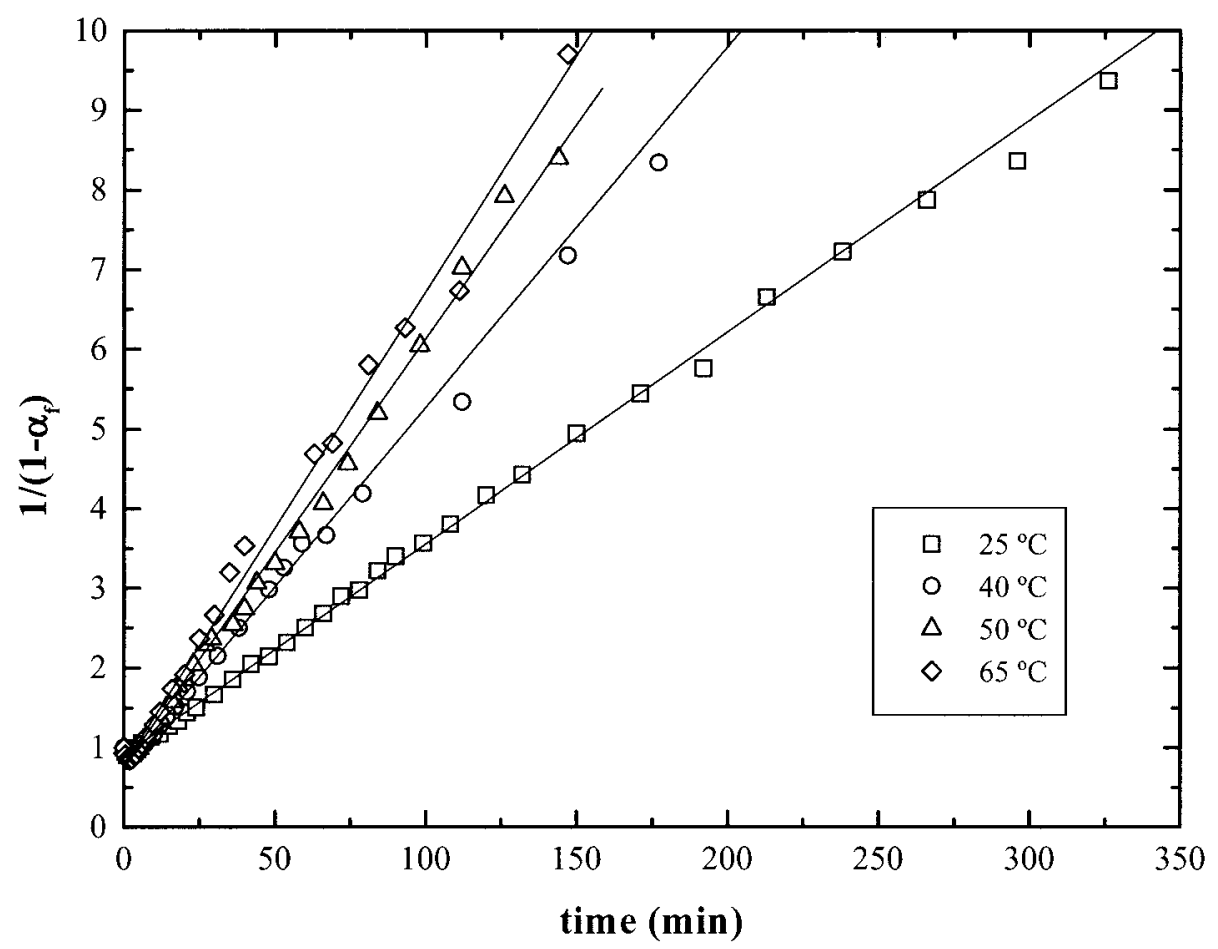

Figure 8 Classical plot for second-order kinetics. $\alpha_{f}$ is the conversion, obtained from the fluorescence of the DNS label, during the curing of PU1096.

these cases, for which the shifts are very small and the fluorometers are not accurate enough to detect changes, the problem is solved by the averaging of the fluorescence band. This method offers, in most cases, plots with very low data dispersion, even when the changes are very small.

The correlation between the first-moment values and conversion percentages measured by FTIR $^{10}$ (Fig.

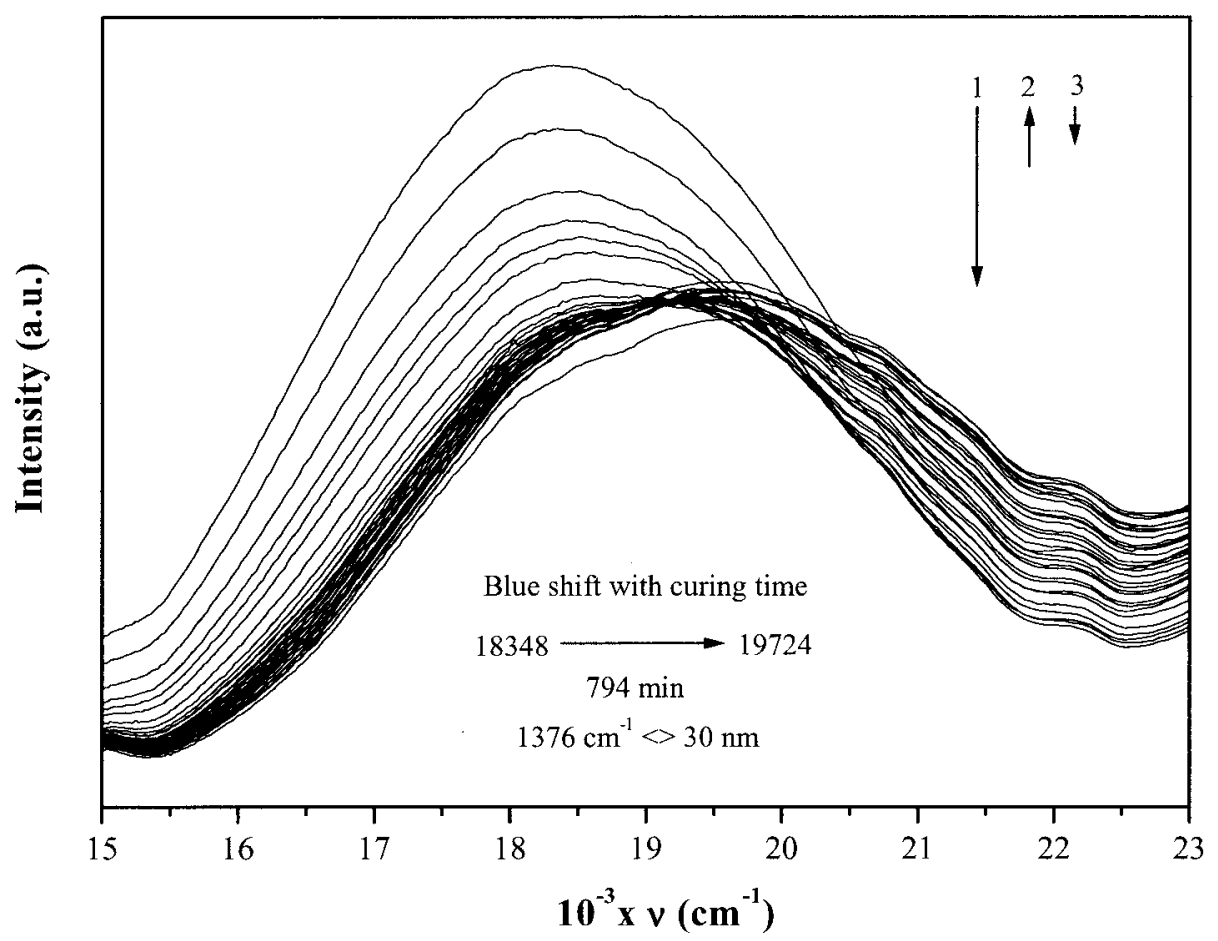

Figure 9 Fluorescence spectra of the DANS label at different curing times for the reaction of PU1096 at $25^{\circ} \mathrm{C}$. 

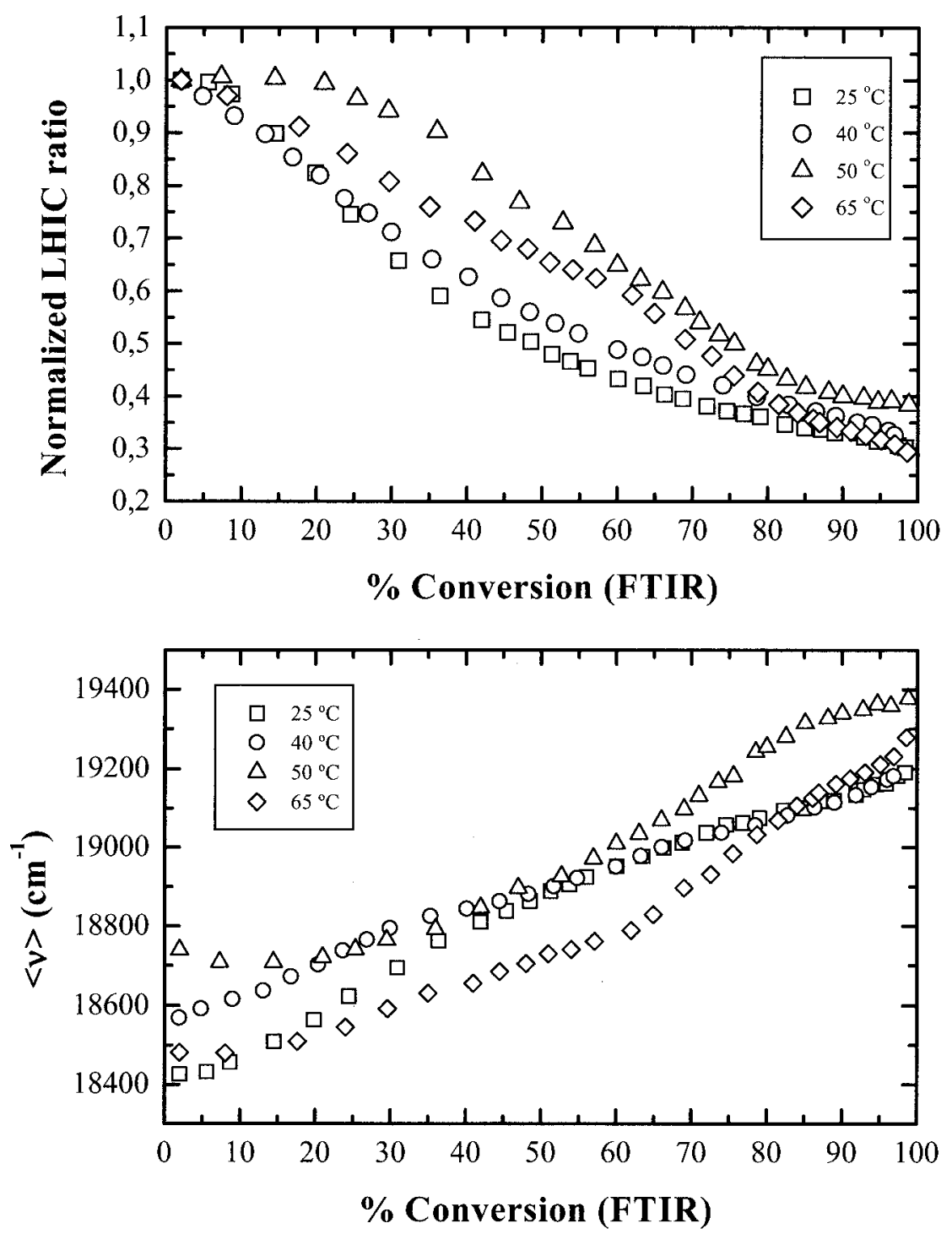

Figure 10 Normalized LHIC ratios (top) and first-moment values (bottom), obtained from the DANS label fluorescence, as a function of the conversion obtained by FTIR for the PU1096 curing process.

7) shows the same results as the normalized LHIC ratios show. Therefore, this method can monitor the curing of PUs at any reaction time, and one could select any of these data processing methods to do the kinetic analysis. In fact, the two parameters studied seem to change even when FTIR conversions are higher than $100 \%$. These results suggest that fluorescence solves the problems of saturation and sensitivity of the classical methods (FTIR and DSC). For the DNS label, we selected the first-moment method, first because of its physical meaning and second because at any temperature there is a direct correspondence between the $\langle\nu\rangle$ value and the chemical conversion.

Taking into account the aforementioned results, to estimate correctly the activation energies in cure processes, we prefer to use the relative extents of cure instead of the absolute values; ${ }^{31}$ for the kinetic analysis, we define $\alpha_{f}$ as follows:

$$
\alpha_{f}=\frac{\nu_{i}-\langle\nu\rangle_{0}}{\langle\nu\rangle_{\infty}-\langle\nu\rangle_{0}}
$$

where $\langle\nu\rangle_{0},\langle\nu\rangle_{t}$, and $\langle\nu\rangle_{\infty}$ are the first-moment values at times $t=0, t=t$, and $t=\infty . \alpha_{f}$ as a function of the PU curing time showed behavior similar to that found with FTIR. ${ }^{10}$

With the classical second-order reaction rate equation for the kinetics of the reactions between NCO and OH groups assumed, the plot of $1 /\left(1-\alpha_{f}\right)$ versus time should yield a straight line (Fig. 8). As can be seen in Figure 8 , the fluorescence data clearly confirm the second-order kinetics with a correlation factor greater than 0.996 . When FTIR and DSC data are used, ${ }^{9,10}$ only part of the curing reaction supports the second-order reaction mechanism. This apparent discrepancy indicates that the classical techniques (FTIR and DSC) are not sensitive enough during the last stages of the 
curing reactions. From the slope of the Arrhenius plot (ln $k$ vs $1 / \mathrm{T}$ ), the apparent activation energy, 16.9 $\pm 3.9 \mathrm{~kJ} / \mathrm{mol}$, has been calculated.

\section{DANS label}

The fluorescence spectra of the DANS label for different curing times when the reaction of PU1096 takes place at $25^{\circ} \mathrm{C}$ are shown in Figure 9 as examples. A broad band can be seen with signals of a background emission that undergoes a blueshift with the curing of PU. For this fluorescence label, the emission intensity continuously decreases as a function of the curing time. An apparent increase in the fluorescence intensity appears during the last stages of the curing. This result was confirmed to be an artifact when the spectra of the labeled sample were subtracted from the fluorescence background of the PU1096. The decrease in the emission intensity with an increase in the viscosity is not a general phenomenon. We interpret this result in terms of one of the two possible nonradiative processes that can occur in the excited state of DANS: ${ }^{32}$ (1) trans-cis isomerization around the stilbene double bond can occur and (2) as the reaction of PU takes place, the number of $\mathrm{OH}$ groups decreases, giving the system a less polar character. The cis isomer is favored in low-polarity solvents, and because it does not fluoresce, a decrease in polarity means a decrease in the fluorescence intensity, as shown in Figure 9. In fact, as curing proceeds, the labeled sample goes from the characteristic yellow color of the trans isomer stilbene to white.

Since the trans-cis isomerization does not affect the energy associated with the transition that gives fluorescence for the DANS label, the use of the LHIC and the first-moment methods are also valid for kinetic studies in the PU1096 system.

Figure 10 shows the correlation between normalized LHIC ratios (top) and the first-moment values (bottom) with the conversion percentages measured by FTIR. Similar observations are found with the DNS label. Nevertheless, when DANS is used, kinetic studies cannot be performed because the LHIC ratios and the $\langle\nu\rangle$ values continuously increase without leveling off at longer curing times. Apparently, there is an important contribution to the blueshift due to high scattering. Therefore, it can be concluded that the nitrostilbene derivatives are not good fluorophores for monitoring the curing processes with large changes in the polarity of the system because of the potential trans-cis isomerization process.

\section{CONCLUSIONS}

The use of the fluorescence response from DNS and DANS labels has been demonstrated to be a good method for studying the conversion of the curing in PUs. The two photophysical parameters used to follow the cure process are the LHIC ratio and the first moment of the fluorescence. Changes even at very high conversions are obtained with respect to FTIR. This suggests that the fluorescence method might solve the problems of sensitivity found with the classical methods (FTIR and DSC).

With the relative variation of the first moment for the DNS label, it was possible to model the kinetics of the PU system and obtain an apparent activation energy of $17 \mathrm{~kJ} / \mathrm{mol}$.

\section{References}

1. Baker, J. W.; Davis, M. M.; Gaunt, J. J Chem Soc 1949, 9, 19.

2. Cawse, J. L.; Standford, J. L.; Still, R. H. Makromol Chem 1984, $185,709$.

3. Sato, M. J Am Chem Soc 1960, 82, 3893.

4. Chorng-Shyan, C. J Appl Polym Sci 1990, 40, 2189.

5. Anzuino, G.; Pirro, A.; Rossi, O.; Friz, L. P. J Polym Sci Polym Chem Ed 1975, 13, 1657.

6. Eceiza, A.; Zabala, J.; Egiburu, J. L.; Corcuera, M. A.; Mondragón, I.; Pascault, J. P. Eur Polym J 1999, 35, 1949.

7. Gambiroza-Jukic, M.; Gomzi, Z.; Mencer, H. J. J Appl Polym Sci 1993, 47, 513.

8. Boufi, S.; Belgacem, M. N.; Quillerou, J.; Gandini, A. Macromolecules 1993, 26, 6706.

9. Shuyan, L.; Vuorimaa, E.; Lemmetyinen, H. J Appl Polym Sci 2001, 81, 1474.

10. Shuyan, L.; Vatanparast, R.; Vuorimaa, E.; Lemmetyinen, H. J Polym Sci Part B: Polym Phys 2000, 38, 2213.

11. Feger, C.; Molis, S. E.; Hsu, S. L.; MacKnight, W. J. Macromolecules 1984, 17, 1830.

12. Michael, J. E.; Anthony, J. R. Polymer 1996, 37, 1351.

13. Mikes, F.; Serrano, B.; González-Benito, J.; Bravo, J.; Baselga, J. Polymer 2002, 43, 4331.

14. Strehmel, B.; Strehmel, V.; Younes, M. J Polym Sci Part B: Polym Phys 1999, 37, 1367.

15. Serrano, B.; Levenfeld, B.; Bravo, J.; Baselga, J. Polym Ing Sci 1996, 36, 175.

16. Mikeš, F.; González-Benito, J.; Baselga, J. Book of Abstracts, XVIII IUPAC Symposium on Photochemistry, Dresden, Germany, July 22-27, 2000.

17. Loutfy, R. O. Macromolecules 1981, 14, 270.

18. Song, J. C.; Sumug, C. S. P. Macromolecules 1993, 26, 4818.

19. Vatanparast, R.; Li, S.; Hakala, K.; Lemmetyinen, H. Macromolecules 2000, 33, 438.

20. Hakala, K.; Vatanparast, R.; Li, S.; Peinado, C.; Bosch, P.; Catalina, F.; Lemmetyinen, H. Macromolecules 2000, 33, 5954.

21. Sun, X.; Sung, C. P. S. Macromolecules 1996, 29, 3198.

22. Yu, J.-W.; Sung, C. S. P. J Appl Polym Sci 1997, 63, 1769.

23. González-Benito, J.; Cabanelas, J. C.; Aznar, A. J.; Vigil, M. R.; Bravo, J.; Serrano, B.; Baselga, J. J Lumin 1997, 72, 451.

24. González-Benito, J.; Cabanelas, J. C.; Vigil, M. R.; Aznar, A. J.; Bravo, J.; Baselga, J. J Fluoresc 1999, 9, 51.

25. Vigil, M. R.; Bravo, J.; Atvars, T. D. Z. ; Baselga, J. Macromolecules 1997, 30, 4871.

26. Song, J. C.; Neckers, D. C. J Polym Eng Sci 1996, 36, 3.

27. Paczkowski, J.; Neckers, D. C. Macromolecules 1992, 25, 548.

28. Li, Y.; Chan, L.; Tyer, L.; Moody, R. T.; Himel, C. M.; Hercules, D. M. J. Am Chem Soc 1975, 97, 3118.

29. González-Benito, J.; Cabanelas, J. C.; Aznar, A. J.; Bravo, J.; Vigil, M. R.; Baselga, J. J Appl Polym Sci 1996, 62, 375.

30. Lackowic, J. R. Principles of Fluorescence Spectroscopy; Plenum: New York, 1986.

31. Vyazovkin, S.; Sbirrazzuoli, N. Macromol Rapid Commun 2000, 21,85 .

32. Lenhart, J. L.; van Zanten, J. H.; Dunkers, J. P.; Zimba, C. G.; James, C. A.; Pollack, S. K.; Parnas, R. S. J Colloid Int Sci 2000, 221, 75 . 